\title{
Beam shaping of multiple laser diodes using a kinoform
}

\author{
Yosuke Koga $^{a^{*}}$, Yuki Misaki ${ }^{a}$, Taiki Nakano ${ }^{a}$, Toshinori Hora ${ }^{a}$, Shiyuan Yang ${ }^{a}$ \\ ${ }^{a}$ Kyushu Institute of Technology, 1-1 Sensui-cho, Tobata-ku, Kitakyushu, Fukuoka, 804-8550, Japan \\ *Corresponding Author: koga@ boss.ecs.kyutech.ac.jp
}

\begin{abstract}
Kinoform is a kind of computer-generated holograms which only has phase modulation. It has high utilization efficiency of illuminating light because a kinoform does not have amplitude modulation. Because of the above advantage of high utilization efficiency, kinoform is desirable as beam shaping element. This study is a location of the basic study to design the kinoform which can shape the laser beams of multiple laser diodes. We inspected the influence for beam shaping of multiple laser diodes without phase difference and discuss the dependence of shaped beam intensity on a ratio of Gaussian radius and range of laser light, the number of laser diodes and the sampling points of the desired shaped beam intensity.
\end{abstract}

Keywords: Kinoform, Multiple laser diodes, Beam shaping.

\section{Introduction}

Laser diodes have merits of compact size and higher luminous efficacy and demerit of low output light power compared to other lasers. It is necessary to use multiple laser diodes or fiber laser, diode bar laser for the applications of laser machining, laser marking and etc. In addition, beam shaping of output light is necessary to increase the quality of application. Computer-generated holograms, especially kinoforms that have high illuminating light efficiency than other computer-generated holograms, are powerful for the beam shaping of laser light, but there is no method to shape multiple laser lights because the phase differences of laser sources are not determined at every power-on time. We suggest a design method for the beam shaping of multiple laser sources of a kinoform using band-unlimited reconstruction to relax the influence of phase differences of laser sources. In this study, we inspected the influence for beam shaping of multiple laser diodes without phase difference as basic study to design the kinoform which can shape the laser beams of multiple laser diodes

\section{Principle of the kinoform}

Figure 1 shows Fourier transformation type kinoform playback optical system. Kinoform is designed from the phase component calculated by inverse Fourier transform of the original image. Since the two-dimensional image which should be reconstructed is intensity distribution, the phase of an original image can be given arbitrarily. Two-dimensional intensity distribution of the original picture image with $N \times N$ pixels which is given by a random phase distribution is represented by $f(x, y)$, the sampled data is represented by $f(m, n)$ and Fourier inverse transform of $f(x, y)$ is represented by the following equation.

$$
\begin{aligned}
F(k, l) & =\frac{1}{N} \sum_{x=-\frac{N}{2}}^{\frac{N}{2}-1} \sum_{y=-\frac{N}{2}}^{\frac{N}{2}-1} f(x, y) \exp \left[-\frac{j 2 \pi}{N^{2}}(k x+l y)\right] \\
& =|F(k, l)| \exp [j \phi(k, l)]
\end{aligned}
$$

The kinoform distribution is represented $W(k, l)$ by replacing the amplitude value $F(k, l)$ of the Fourier components with the constant value, quantizing phase components to the quantized number of $\mathrm{L}$ values. The kinoform distribution $W(k, l)$ is represented by the following equation.

$$
W(k, l)=A(k, l) \exp [j \Phi(k, l)]
$$

Intensity distribution of the reconstructed image is represented by the following equation. $G_{s}(k, l)$ represents the Gaussian distribution of the laser beam. 


$$
\begin{aligned}
\left|F^{\prime}(m, n)\right|^{2} & =\mid \sum_{x=-\frac{N}{2}}^{\frac{N}{2}-1} \sum_{y=-\frac{N}{2}}^{\frac{N}{2}-1}\left\{G_{s}(k, l)\right. \\
& \left.\times W(k, l) \exp \left[-\frac{j 2 \pi}{N^{2}}(m k+n l)\right]\right\}\left.\right|^{2}
\end{aligned}
$$

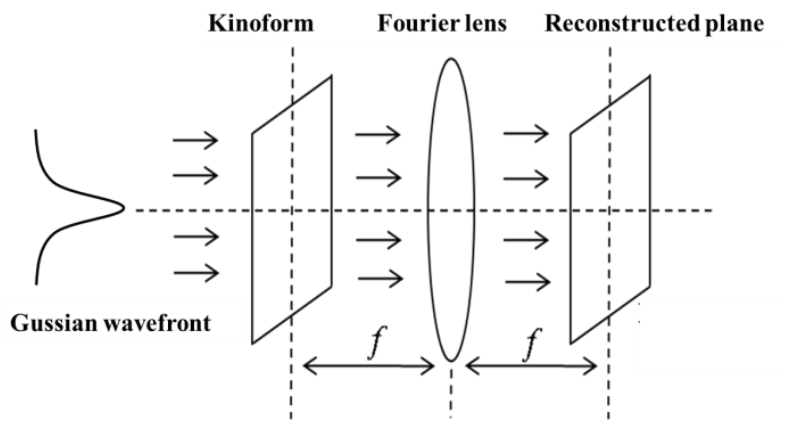

Fig. 1. Fourier transformation type

\section{Iterative dummy area method}

The error is reconstructed in reconstructed image of the kinoform because it does not have amplitude modulation Iterative dummy area method is an algorithm which reduces this error. The flow of this iterative dummy area method is shown in Fig. 2. To reduce error is possible by adding the dummy area of the initial value 0 to the surroundings of an original image, use this picture as input picture and by using the amplitude / phase flexibility of a dummy area.

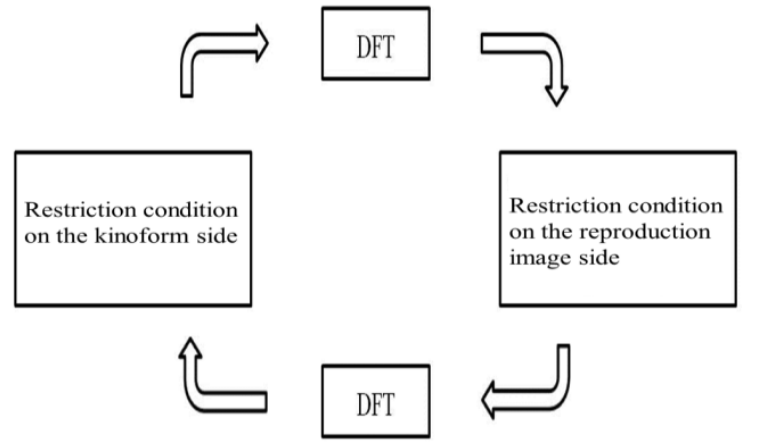

Fig. 2. Iterative dummy area method

As a restriction condition on the kinoform side, the obtained amplitude distribution is replaced by the amplitude distribution of the Gaussian distribution. As a restriction condition on the reconstructed image side, the obtained amplitude distribution is replaced by the amplitude of the original picture image. Fig. 3 shows the original image add a blank space. And, the phase of the original image is random phase.

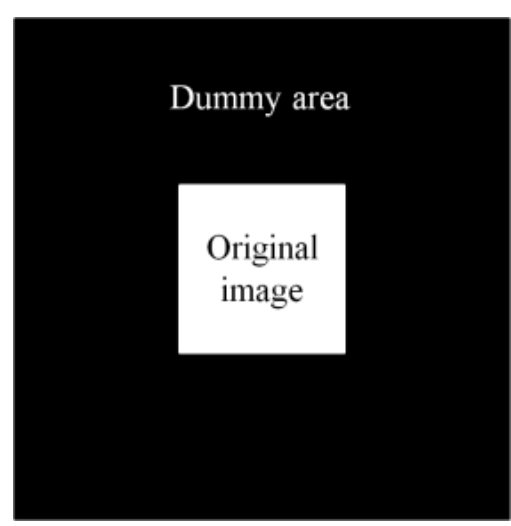

Fig. 3. Dummy area

\section{Simulation and results}

Laser light is the Gaussian distribution in this simulation. Multiple laser lights are placed in square. Phase difference of multiple laser lights are a zero in this simulation. Gaussian radius of a laser light " $R$ " is represented by the following equation.

$$
R=N / A / S
$$

The range of one laser light is limited $T \times T$ [pixel] in this simulation and the range of the laser light " $T$ " is represented by the following equation.

$$
T=N / A
$$

" $N \times N$ " represents Dummy area. " $A \times A$ " represents the number of laser lights. " $S$ " represents the parameter to decide a ratio of Gaussian radius and range of laser light. Figure 4 shows the positional relationship of the laser lights to input to the kinoform side when " $S$ " is 4 .

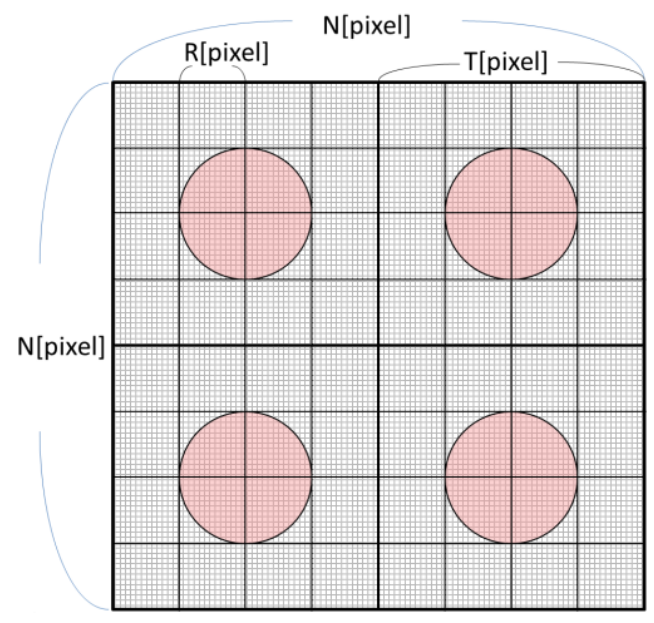

Fig. 4. Positional relationship of the laser lights

Figure 5 shows Fourier transformation type kinoform playback optical system of multiple lasers. 


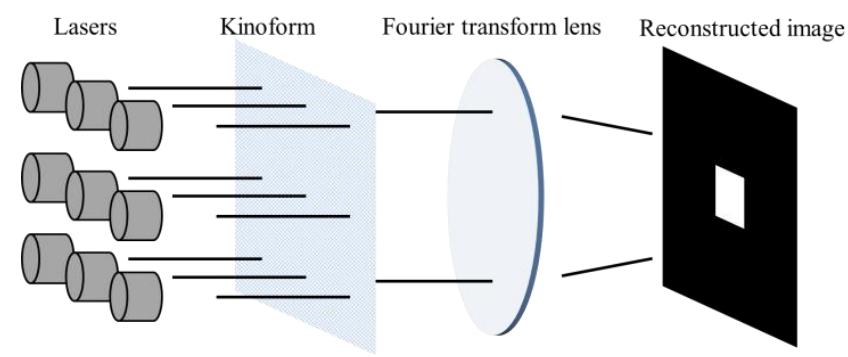

Fig. 5. Fourier transformation type kinoform playback optical system of multiple lasers

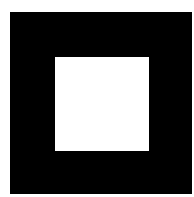

Fig. 6. Original image

We use a square image of Fig. 6 as the original image.

At first, we inspected the optimal value of " $S$ ".

The condition of simulation 1 is as follows:

- Original image

$64 \times 64$ [pixel]

- Initial phase Random phase

- Dummy area

- Number of iteration $512 \times 512$ [pixel]

- Number of laser light 1000 times $8 \times 8$

- Parameter : " $S$ "

Figure 7 shows the result of simulation 1.

The more " $S$ " increases, the more noises are reconstructed in the dummy area around the original image in Fig. 7. In the case " $S$ " is 5 or 6 , noise is big. When " $S$ " is 2 or 3 , the noises decrease but utilization efficiency of illuminating light is low because the range of laser lights is limited. The result of simulation 1 shows that the value is the optimal when " $S$ " is 4.

Next, we inspected the effect of samples of original image.

The condition of simulation 2 is as follows:

- Original image

$32 \times 32$ [pixel], $64 \times 64$ [pixel], $128 \times 128$ [pixel]

- Initial phase Random phase

- Dummy area $512 \times 512$ [pixel]

- Number of iteration 1000 times

- Number of laser light $4 \times 4,8 \times 8$, $16 \times 16,32 \times 32$

- Parameter : " $S$ "

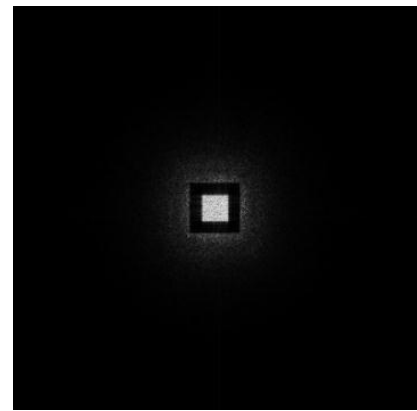

(a) $S=2$

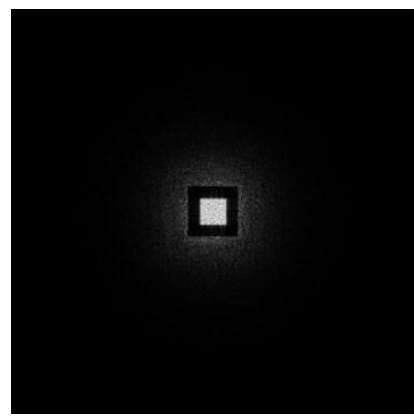

(b) $S=3$

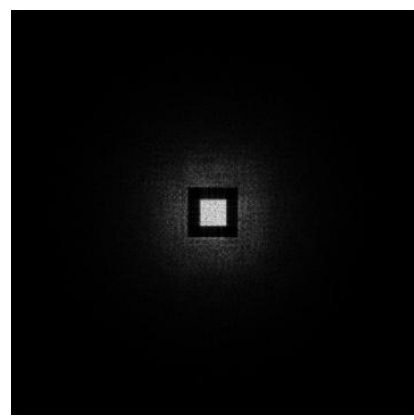

(c) $S=4$

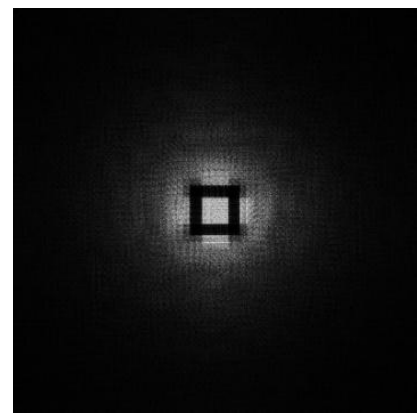

(d) $S=5$

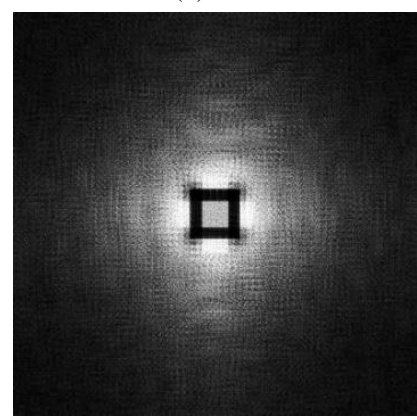

(e) $S=6$

Fig. 7. Result of simulation 1

Figures 8-11 show the results of simulation 2 . 


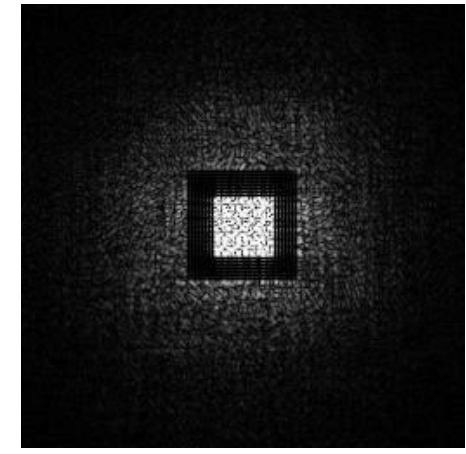

(a) Original image: $32 \times 32$ [pixel]

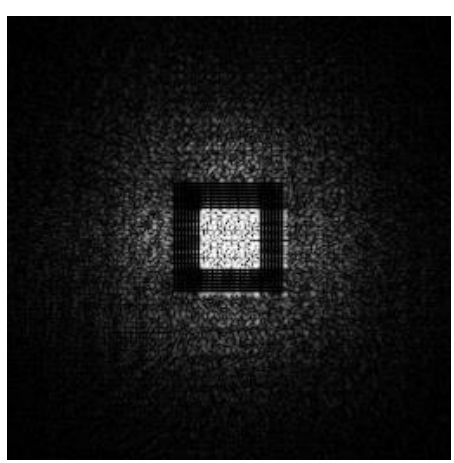

(a) Original image: $32 \times 32$ [pixel]

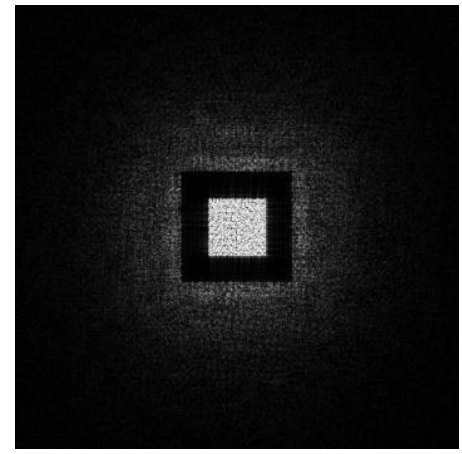

(b) Original image: $64 \times 64$ [pixel]

Fig. 8 . The number of laser light is $4 \times 4$

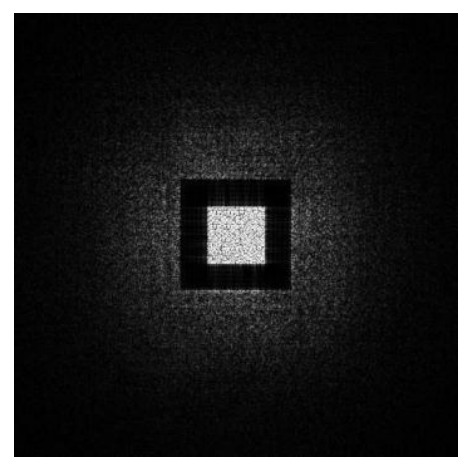

(b) Original image: $64 \times 64$ [pixel]

Fig. 9. The number of laser light is $8 \times 8$

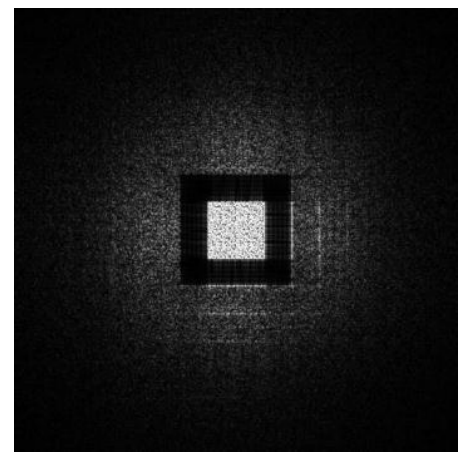

(b) Original image: $64 \times 64$ [pixel]

(a) Original image: $32 \times 32$ [pixel]

Fig. 10. The number of laser light is $16 \times 16$

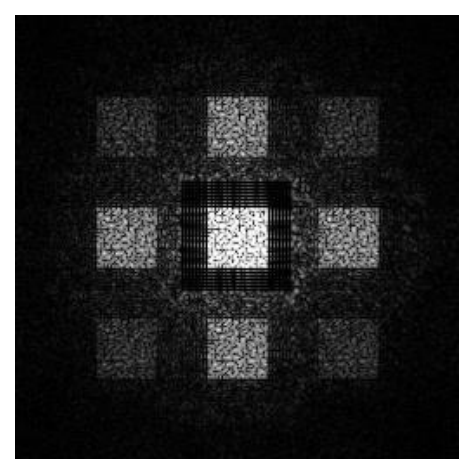

(a) Original image: $32 \times 32$ [pixel]

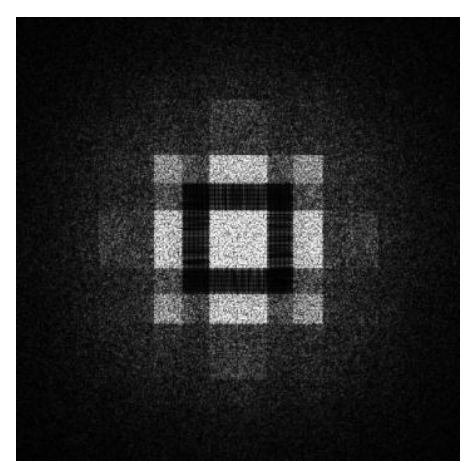

(b) Original image: $64 \times 64$ [pixel]

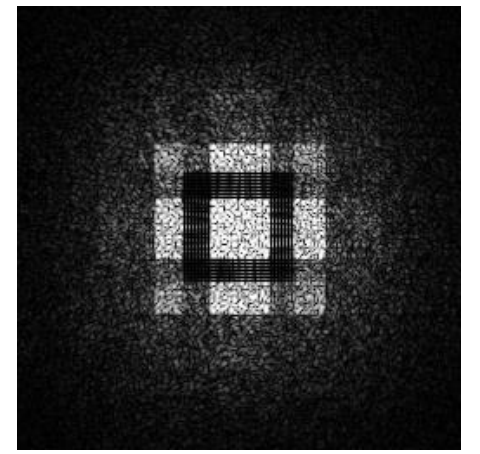

Fig. 11. The number of laser light is $32 \times 32$

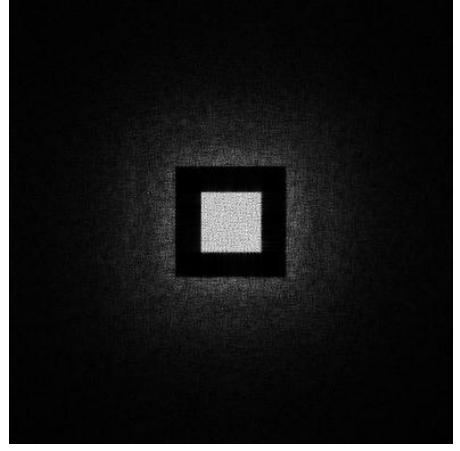

(c) Original image: $128 \times 128$ [pixel]

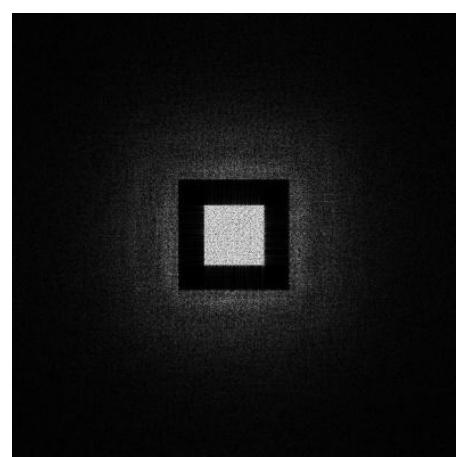

(c) Original image: $128 \times 128$ [pixel]

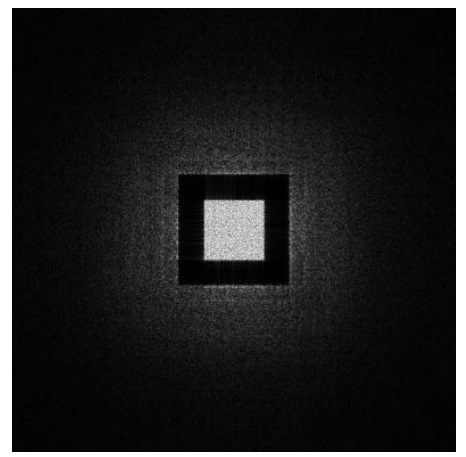

(c) Original image: $128 \times 128$ [pixel]

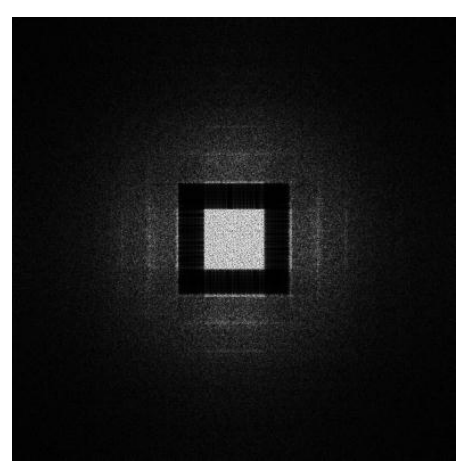

(c) Original image: $128 \times 128$ [pixel] 
Noises are reconstructed in the dummy area around the original image in Figs. 8-11. In comparison of Figs. 8-11, the more the number of laser lights is large, the more noises are constructed in the dummy area around the original image. In the case the sampling points of original image is large, noise is small level even if the number of laser lights is large.

\section{Conclusions}

In this study, the basic dependences of reconstruction noise are carried out on a ratio of Gaussian radius and range of laser light, the number of laser diodes and the sampling points of original image. In case of designing the kinoform which can shape the laser beams of multiple laser diodes, it should be noted about the above. As a future study, we will study beam shaping of multiple laser lights with phase difference of a kinoform.

\section{References}

(1) H. Aagedal, M. Schmid, T. Beth, S. Teiwes, and F. Wyrowski: "Theory of speckles in diffractive optics and its application to beam shaping", J. Mod. Opt., No.43, 1409-1421, 1996

(2) Fred M. Dickey: "Laser beam shaping", Optics and photonics news, Vol. 14, No. 4, 30-35, 2003

(3) N. C. Gallagher and B. Liu: "Method for computing kinoforms that reduces image reconstruction error.", Applied Optics, Vol. 12, No. 10, 2328-2335, 1973

(4) L. B. Lesem, P. M. Hirsch, and J. A. Jordan: "The kinoform: a new wavefront reconstruction device", IBM Journal of Research and Development, Vol. 13, No. 2, 150-155, 1969 